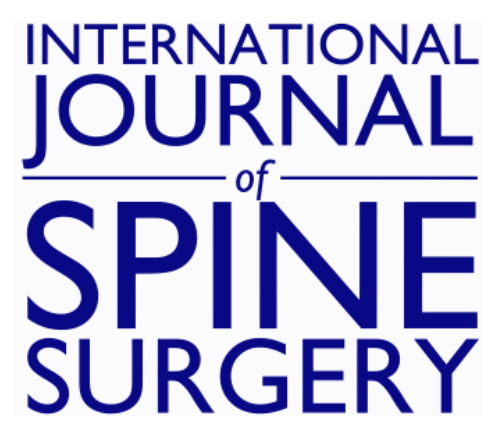

\title{
Endoscopic Transforaminal and Lateral Recess Decompression After Previous Spinal Surgery
}

\author{
KAI-UWE LEWANDROWSKI \\ Int J Spine Surg 2018, 12 (2) 98-111 \\ doi: https://doi.org/10.14444/5016 \\ http://ijssurgery.com/content/12/2/98
}

This information is current as of April 26, 2023.

Email Alerts Receive free email-alerts when new articles cite this article. Sign up at:

http://ijssurgery.com/alerts

The International Journal of Spine Surgery

2397 Waterbury Circle, Suite 1,

Aurora, IL 60504, Phone: +1-630-375-1432

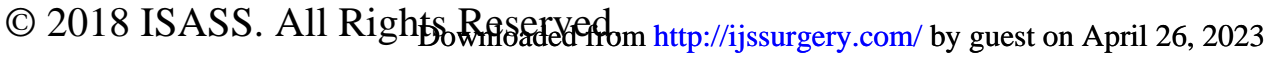




\title{
Endoscopic Transforaminal and Lateral Recess Decompression After Previous Spinal Surgery
}

\author{
KAI-UWE LEWANDROWSKI, MD \\ Center for Advanced Spine Care of Southern Arizona, Tucson, Arizona
}

\begin{abstract}
Background: Leg pain and back pain after lumbar laminectomy and spinal decompression fusion surgery are common and often related to persistent lumbar foraminal or lateral recess stenosis. Although persistent symptoms often stem from incomplete decompression during the primary index surgery, recurrent symptoms may also be the result of intervertebral cage subsidence due to loss of intervertebral and neuroforaminal height.

Objective: The aim of this study was to investigate the feasibility of using the outpatient transforaminal decompression procedure as an alternative to inpatient open procedure in revision decompression surgery, with the intent of minimizing the incidence of perioperative and postoperative surgical complications while reducing both direct and indirect costs of surgical treatment, shortening time to patient postoperative narcotic independence, and shortening time of patient return to daily activities.
\end{abstract}

Methods: A total of 48 patients with conclusive diagnostic imaging and interventional workup underwent endoscopic transforaminal and lateral recess decompression for both persistent or recurrent leg and/or low back pain following previous lumbar laminectomy (22 patients) or decompression fusion surgery (26 patients). In addition to radiographic studies, patients were followed for a minimum of 2 years postoperatively, and clinical outcomes were evaluated with the visual analog scale (VAS), Oswestry Disability Index (ODI), and modified Macnab criteria.

Results: At final follow-up, patients with single- and 2-level prior surgeries reported an average ODI reduction following their secondary surgery of $44.6 \%$, with an average final score of 14.8. Less favorable ODI score reductions following secondary surgery $(23.8 \%)$ were reported by patients who had more than one or a complex prior multilevel surgery. According to the modified Macnab criteria, "excellent" and "good" results were obtained with the secondary surgery in $79.1 \%$ ( 38 of 48 ) of patients with no more than a single 1- or 2-level prior lumbar surgery. The mean VAS score decreased from $7.7 \pm 1.8$ preoperatively to $2.3 \pm 1.1$ at final follow-up $(P<.01)$. Fair and poor results with the secondary surgery were seen in $20.9 \%$ (10 of 48 ) of patients with several prior surgeries or complex multilevel previous lumbar surgeries. The level distribution for secondary surgery was as follows: L4-5 segment (26 levels; $54.1 \%$ ), L5-S1 (14 levels; 29.2\%), L3-4 (7 levels; 14.6\%), and the L2-3 level (1 level; 2.1\%). Postoperative complications were limited to irritation of the dorsal root ganglion, which occurred in $25 \%$ (12 of 48 ) of patients. There were no wound infections, nerve root injuries, foot drop, or admissions to a hospital for further postoperative care. All patients with "excellent" and "good" outcomes measured by modified Macnab criteria, who were working before and after the primary and secondary surgeries ( 27 of 38), reported earlier return to work after the endoscopic outpatient surgery (2.6 \pm 0.8 weeks) than with the prior inpatient open spinal surgery $(8.1 \pm 4.5$ weeks). Based on the 2012 Medicare fee schedule for professional fees, direct costs were $40.6 \%$ and indirect costs were $37.1 \%$ lower with the secondary endoscopic surgery compared with primary open surgery.

Conclusions: Transforaminal decompression is an effective alternative to open revision lumbar spinal surgery to treat symptomatic spinal stenosis after previous lumbar surgery in patients with persistent or recurrent leg and low back pain. It can be safely done in an outpatient setting, while realizing savings in direct and indirect costs.

Minimally Invasive Surgery

Keywords: foraminal lateral recess stenosis, endoscopic decompression, failed lumbar surgery

\section{INTRODUCTION}

Ongoing or new-onset leg and back pain following an initial period of pain relief after previous lumbar laminectomy or decompression fusion surgery is not an uncommon scenario. Contributing factors include poor psychosocial coping skills, narcotic dependence, presence of work-related injury, incomplete decompression during the primary index surgery, progression of the degenerative disease process with development of instability following laminectomy, and failure of spinal implants in cases of combined instrumented lumbar decompression fusion surgery. With increasing 
scrutiny on the indications and outcomes of lumbar spinal surgery when the original index procedure has failed, there has been a push for use of more evidence-based treatments and a necessity for costbenefit assessment of any planned subsequent surgical treatment.

Patients, insurance providers, and governmental review boards are increasingly aware of the high cost of lumbar spinal surgery, and that in some patients it may propagate the need for additional spinal surgery soon after the original index procedure in order to treat symptoms related to postlaminectomy-, and failed back syndrome, instability, or adjacent segment disease. At the same time, there is a growing demand for these lumbar surgical procedures because of an increasing aging baby boomer population that has spinal stenosisrelated pain syndrome. The challenge is: How will physicians provide high-value health care to those in need without increasing the rolls of patients with continued postoperative problems?

With the advent of minimally invasive spinal procedures, including endoscopic spinal surgery, hospital admissions have decreased significantly and many of the surgical procedures, as well as the preoperative and postoperative patient management, are now being done in an ambulatory surgery center or office setting. The implication is that the use of a surgical technique that does not require extensive muscle stripping or exposure of neural elements after resection of the posterior elements of the lumbar spine is associated with a lower incidence of short- and long-term postoperative problems. The aim of this study was to simply investigate whether a transforaminal decompression procedure can adequately address persistent or new onset of sciatica-type leg and low back pain symptoms following previous lumbar spinal surgery, while reducing both direct and indirect costs of surgical treatment and shortening time to patient postoperative narcotic independence and return to daily activities.

\section{MATERIALS AND METHODS}

In the clinic of the author of the present study, there is an outpatient spinal surgery program for the treatment of lumbar herniated disc and spinal stenosis. The results presented here are based on a retrospective review of patients who were seen from 2010 through 2013. Patients were worked up diagnostically by reviewing prior referrals of con- sulting physicians, assessing pain management and interventional care, and integrating the information into clinical pathways to determine what additional diagnostic study or nonoperative treatment measures may be appropriate prior to considering surgical treatment. Patients were seen for lumbar radiculopathy, with and without claudication, and low back pain.

\section{Patient Population}

All patients in this series provided informed consent. Patients were selected from a group of 258 consecutive patients seen in clinic who underwent endoscopic foraminotomy and microdiscectomy at 289 levels from 2010 through 2013. Ultimately, 48 of the 258 patients had undergone previous lumbar spinal surgery. In this subgroup of 48 of 258 patients, the mean follow-up was 37 months, ranging from 24 to 58 months at the time this study was concluded. The criteria for inclusion were: (1) clinical signs of unilateral lumbar monoradiculopathy, dysesthesia, and decreased motor function; (2) imaging evidence of foraminal or lateral recess stenosis (criteria described below) demonstrated on preoperative magnetic resonance imaging (MRI) and computed tomography (CT) scans; (3) unsuccessful nonoperative treatment, including physical therapy, and transforaminal epidural steroid injections for at least 12 weeks; and (4) an age of 35-85 years. Patients exhibiting pain syndromes involving more than 1 dermatome or had bilateral symptoms, or showed segmental instability on preoperative extension flexion radiographs, or had severe central stenosis (less than 100 $\mathrm{mm}^{2}$ ), or both, were excluded from this study. ${ }^{1}$ Inclusion/exclusion criteria were used with the intent of minimizing the effect of other confounding factors. In addition, maximizing patient's functional outcomes prior to considering further surgical treatment, particularly in patients whose prior open lumbar surgery had been performed less than 1 year from the time they presented to clinic for consultation and additional treatment, was considered prudent. This population of patients was also excluded from the study. These patients received further support and interventional care, and in some cases were referred to pain management.

The average age of the 48 of 258 patients with prior lumbar spinal surgery was $54.3 \pm 11.8$ years; sex distribution was as follows: 29 patients were female and 19 patients were male. Of these 48 

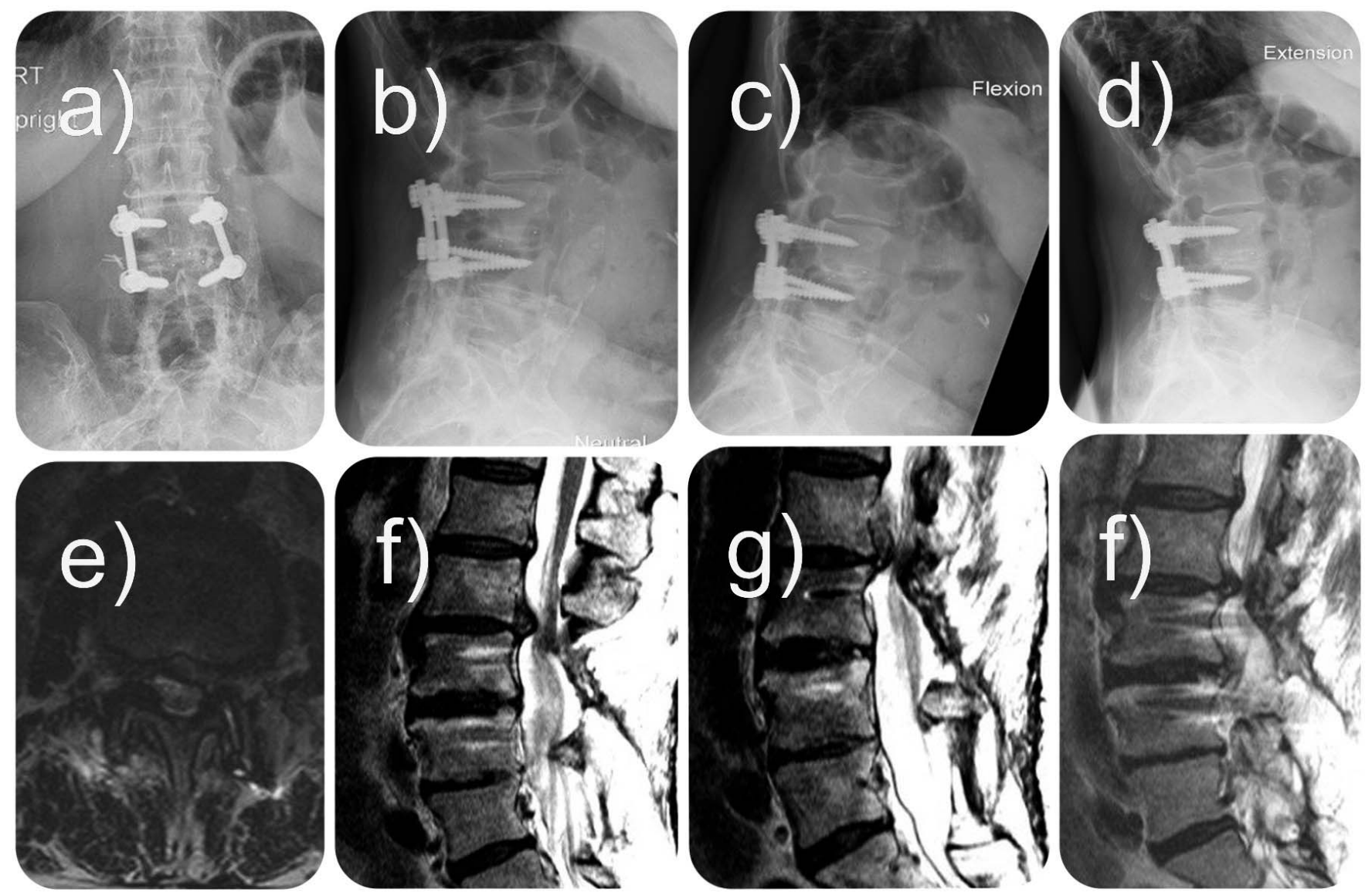

Figure 1. Plain film studies (a-d) and magnetic resonance imaging scans (e-h) of a 76-year-old female patient who underwent previous laminectomy followed by transforaminal interbody fusion (TLIF) at L3-4 years after her previous laminectomy. She presented with new onset of left-side inner thigh pain and neurogenic claudication symptoms 3 years following her TLIF. This patient was not interested in more open spinal surgery of any sort but opted for outpatient transforaminal decompression at L2-3 on the left side to treat the symptoms stemming from the adjacent segment disc herniation and resulting lateral recess stenosis surgically. She went home within an hour from surgery and her symptoms completely resolved immediately postoperatively. Her final follow-up visit was 30 months after secondary endoscopic outpatient decompression; she remained pain free.

patients, 22 had undergone prior laminectomy and 26 underwent decompression fusion surgery with interbody fusion cages and pedicle screws. Of the latter group, 16 patients had a single-level surgery (Figures 1 and 2), another 7 patients had a 2-level surgery, and the remaining 3 patients had multilevel fusion surgeries (Figures 3 and 4). In the laminectomy group, prior single-level surgery had been performed in 14 patients, whereas 5 patients had a 2-level lumbar laminectomy decompression. In the remaining 3 patients of this subgroup, a 3-level laminectomy had been performed prior to the onset of recurrent symptoms, which prompted the referral for another consultation with a spine surgeon (Table 1). Of the 48 patients, 12 were treated by the author; of these 12 patients, 7 had undergone prior lumbar laminectomy and 5 had undergone either prior lumbar laminectomy and/or fusion surgery. The remaining 36 patients were referred with prior surgeries. All of the 48 patients had undergone their previous lumbar spinal surgery in a hospital setting. The average postoperative time that elapsed from the inpatient index surgery to the outpatient endoscopic revision surgery was $3.7 \pm 2.4$ years, ranging from 1.1 to 7.8 years. Most of the patients (37 of 48) complained of recurrent symptoms after some time of pain relief. The remaining 11 patients had some but never complete

Table 1. Patients by prior surgery $(n=48)$.

\begin{tabular}{lc}
\hline Type of Prior Surgery & No. of Patients \\
\hline Lumbar laminectomy & \\
$\quad$ Single-level laminectomy & 14 \\
Two-level laminectomy & 5 \\
Three-level laminectomy & 3 \\
Subtotal & 22 \\
Laminectomy fusion with interbody cages & \\
Single-level fusion & 16 \\
Two-level fusion & 7 \\
Three-level fusion & 3 \\
Subtotal & 26 \\
Total & 48 \\
\hline
\end{tabular}



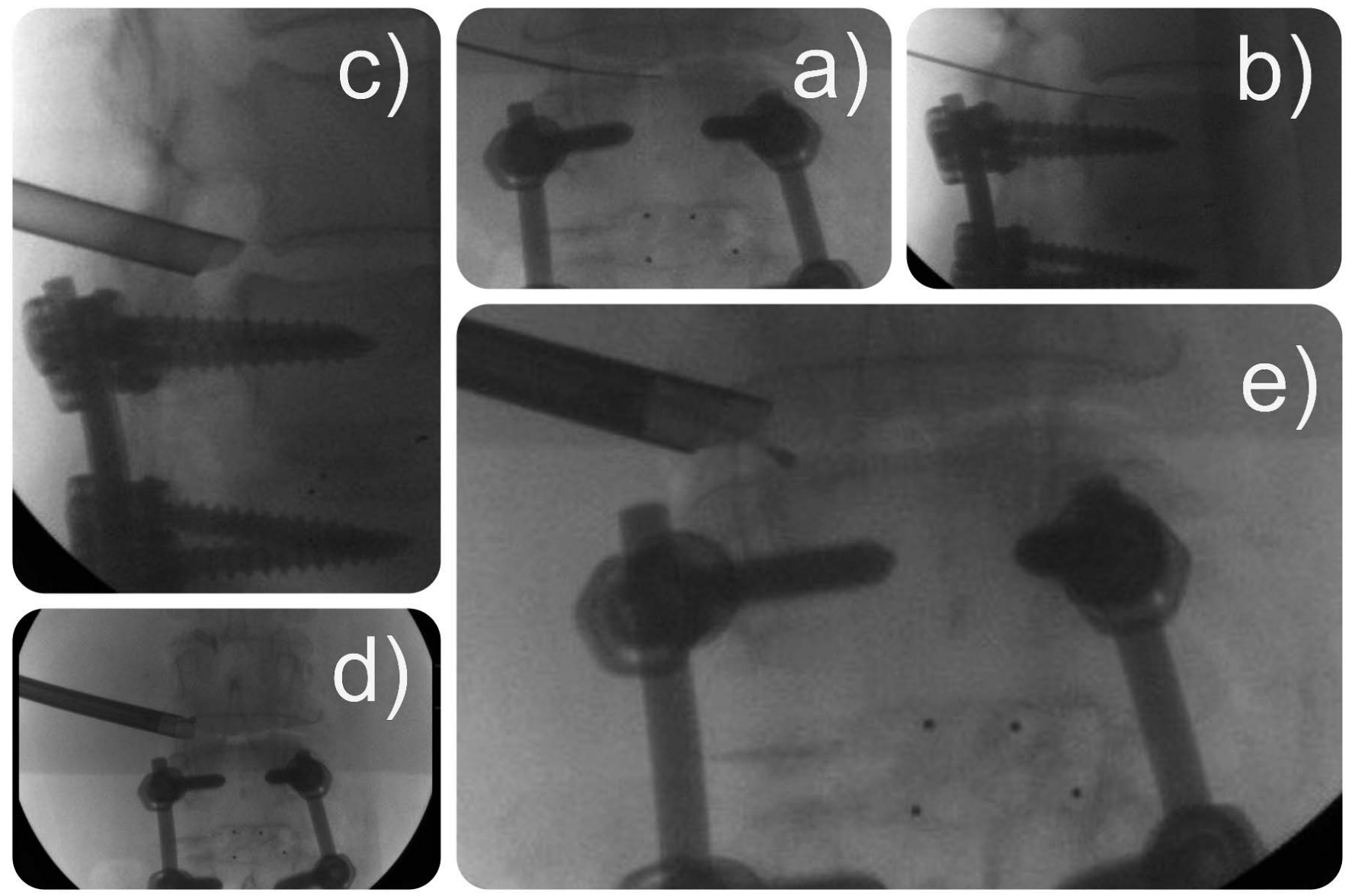

Figure 2. Intraoperative fluoroscopy images of a 76-year-old female patient who underwent previous laminectomy followed by transforaminal interbody fusion at L3-4 years after her previous laminectomy. ( $\mathrm{a}$ and b) Placement of an 18-G, 8-inch guide wire. (c and d) Placement of the working cannula. (e) Placement of the flexible palpation probe into the lateral recess to check for free fragments and to assess the decompression.

pain relief from the initial index procedure (Table 2).

\section{Preoperative Workup and Clinical Follow-Up}

Radiographs, MRI, and CT images were obtained preoperatively in all surgical patients. Postoperatively, CT images were taken if the patient showed no improvement of clinical symptoms after a minimum of 6 weeks after surgery.

Patients returned for clinical follow-up at 6 weeks postoperatively, and at 3,6,12, and 24 months. At

Table 2. Onset of recurrent symptoms $(n=48)$.

\begin{tabular}{lc}
\hline Patients & No. (\%) \\
\hline Initial pain relief from primary surgery & $37(77.1)$ \\
Return of symptoms within 6 mo & $9(46.9)$ \\
Return of symptoms within 1 y & $18(59.0)$ \\
Return of symptoms within 2 y & $8(9.2)$ \\
Return of symptoms within 4 y & $5(13.0)$ \\
Return of symptoms within 8 y & $2(3.8)$ \\
Subtotal & $37(77.1)$ \\
Incomplete pain relief from primary surgery & $11(22.9)$ \\
Total & $48(100)$ \\
\hline
\end{tabular}

24 months, the clinical follow-up on the patient group of 258 was $93 \%$. After the 2-year follow-up appointment, patients were seen on an annual or biannual basis. The long-term follow-up of the 48 patients at 2 years was complete; however, followup was less reliable and available after 3 years $(89 \%)$ and after 4 years $(72 \%)$ postoperatively. Results reported herein, therefore, were computed from data obtained at 2-year follow-up. Primary clinical outcome measures showed reductions in the visual analog score (VAS) for leg pain ranging from no pain (0) to worst pain (10) and the Oswestry Disability Index (ODI) using the Macnab criteria. ${ }^{2}$ Briefly, follow-up results were classified as "excellent" if the patient had little pain and returned to desired activities with few limitations. Outcomes were classified as "good" if the patient reported occasional pain or dysesthesias for daily activities with minor restrictions, and did not need any pain medication. Patients were assigned to 1 of the 2 remaining categories if their pain improved somewhat but they continued to need pain medication 

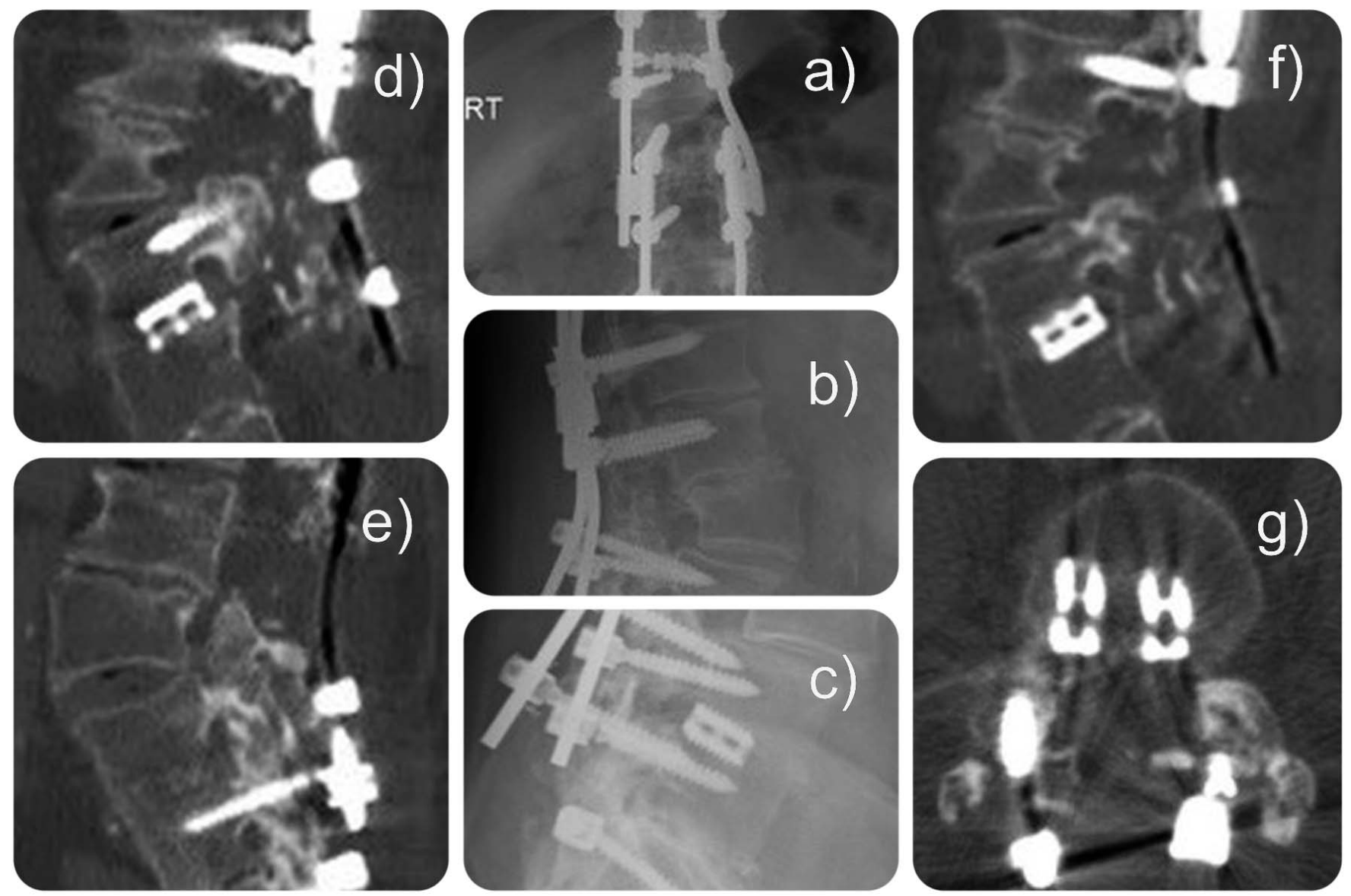

Figure 3. Plain film studies $(\mathrm{a}-\mathrm{c})$ and computed tomography scans $(\mathrm{d}-\mathrm{g})$ of a 69 -year-old male patient. Patient underwent multiple previous lumbar surgeries and last pedicle subtraction osteotomy with residual foraminal and lateral recess stenosis at L4-5 with a slightly posteriorly translocated interbody fusion cage (shaded area in e-g), with some bone formation posteriorly to the cage (shown in f). Patient presented with persistent anterolateral thigh and calf pain and neurogenic claudication symptoms and failed interventional and supportive care. An outpatient transforaminal decompression at L4-5 on the symptomatic left side was performed in this complex situation, where postoperative complications because of an open revision surgery would have been likely. This patient went home within an hour from the outpatient surgery center and reported complete resolution of symptoms at 25 months postoperatively.

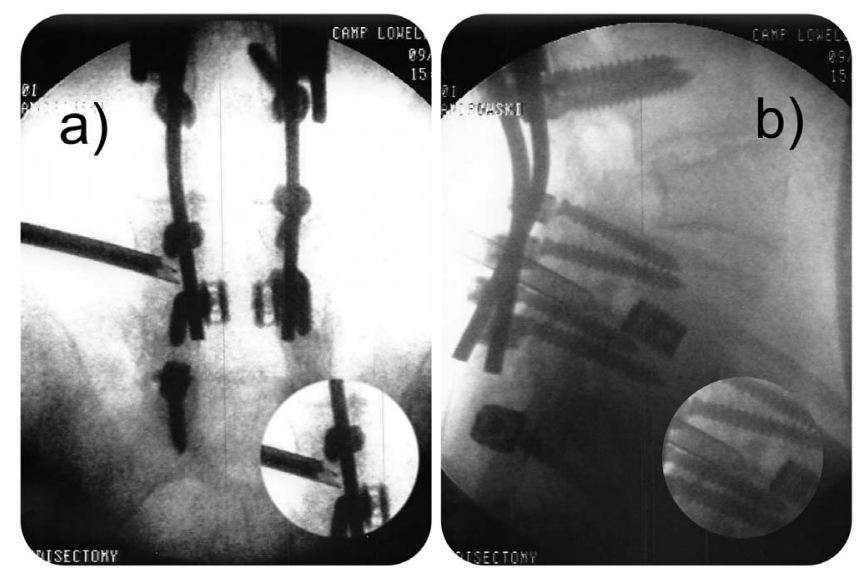

Figure 4. Intraoperative fluoroscopy films (a and b) of a 69-year-old male patient. Patient underwent multiple previous lumbar surgeries and last pedicle subtraction osteotomy with residual foraminal and lateral recess stenosis at L4-5 and with a slightly posteriorly translocated interbody fusion cage. His symptoms of persistent anterolateral thigh and calf pain and neurogenic claudication were treated with an outpatient transforaminal decompression at L4-5. (a) The working cannula is placed under the fusion rod and in between pedicle screws. (b) Access to the lateral recess. ("fair"), or if their function worsened and they needed additional surgery to address their symptoms ("poor").

\section{Radiologic Classification of Foraminal Stenosis}

Classification by Lee et al. ${ }^{3}$ of foraminal and lateral recess stenosis was used to define the location of the offending pathology within the neuroforamen by dividing it from medial to lateral into entry (dura to pedicle; zone 1), middle (medial pedicle wall to center pedicle; zone 2), and exit (center pedicle to lateral border of the facet joint; zone 3) zones. Foraminal and lateral recess stenosis were stratified according to the main offending pathology: extruded herniated disc, disc bulge, and disc bulge with concomitant bony stenosis. Disc herniations were further classified as upward or downward migrated or centered on this disc space using the 4-zone classification by Lee et al. ${ }^{4}$ In the entry zone, Lee et 
al. ${ }^{3}$ described hypertrophy of the superior articular facet as the predominant pathology. In the middle zone, it was often due to an osteophytic process underneath the pars interarticularis, and in the exit zone it was often due to a subluxed and hypertrophic facet joint. ${ }^{3}$ In addition, the intervertebral disc degeneration process with disorganization of its biologic structure was ranked with an MRI-based grading system published by Pfirrmann et al. ${ }^{5}$ Degenerative intervertebral disc process at each surgical level was classified as follows:

- Grade I: Disc is homogeneous, with bright, hyperintense white signal intensity and normal disc height.

- Grade II: Disc is inhomogeneous but keeps the hyperintense white signal. Nucleus and annulus are clearly differentiated, and a gray horizontal band could be present. Disc height is normal.

- Grade III: Disc is inhomogeneous, with intermittent gray signal intensity. Distinction between nucleus and annulus is unclear. Disc height is normal or slightly decreased.

- Grade IV: Disc is inhomogeneous, with a hypointense dark gray signal intensity. There is no distinction between the nucleus and annulus. Disc height is slightly or moderately decreased.

- Grade V: Disc is inhomogeneous, with hypointense black signal intensity. There is no difference between the nucleus and annulus. The disc space is collapsed.

The height of the posterior intervertebral disc and lumbar foramina was evaluated according to Hasegawa et al., ${ }^{6}$ who described a lumbar neuroforaminal height of $15 \mathrm{~mm}$ or more as normal, with reduced posterior intervertebral disc height of 3 to $4 \mathrm{~mm}$ as suggestive of spinal stenosis. Preoperative sagittal and axial MRI and CT images were used to assess the location and extent of foraminal stenosis. Only patients with stenotic lesions (whether due to bony stenosis or extruded disc herniation or contained disc bulge) producing a neuroforaminal width of 3 $\mathrm{mm}$ or less on the sagittal MRI and CT cuts or lateral recess height of $3 \mathrm{~mm}$ or less on the axial MRI and CT cuts were included in this analysis.

A similar analysis of neuroforaminal height and width was used to describe stenotic lesions after instrumented spinal fusion with or without interbody fusion cages, or with or without posterolateral fusion. In the latter, nerve root entrapment was

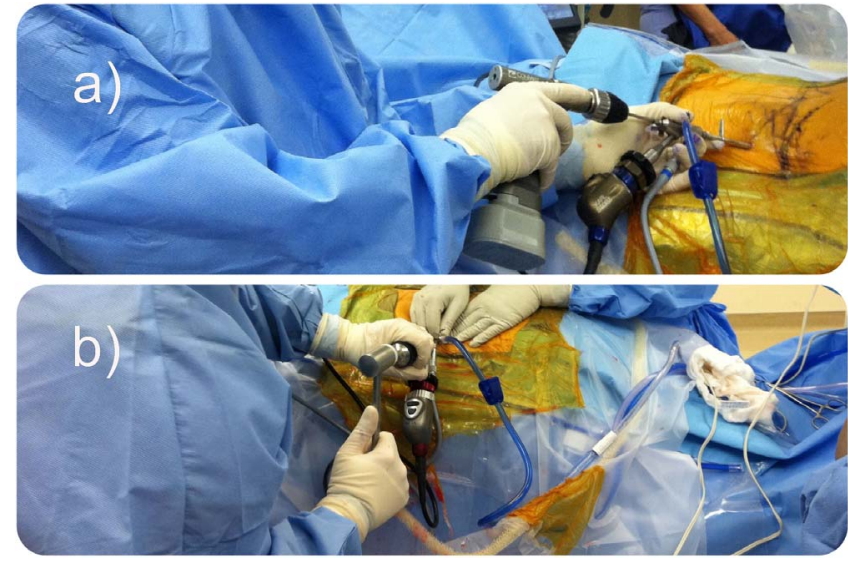

Figure 5. The foraminal drill (a) and chisel (b) shown can be advanced directly to the inner working channel. The drill is attached to a power driver and can be used in forward and reverse directions. It is most suitable for expansile foraminoplasty around the inferior pedicle.

typically caused by bony overgrowth from the posterolateral fusion mass, with predominant encroachment of the exiting nerve root within the fusion or at an adjacent level (Figure 3 and Supplemental Video 1; http://www.ijssurgery.com/ lookup/suppl/doi:10.14444/5016/-/DC1/Video1. mp4). In the former, neural element compression typically resulted from posterior dislocation or subsidence of an interbody fusion cage, both of which were observed to produce impingement of the traversing nerve root either by direct anterior-toposterior displacement of the nerve root or by reactive bone overgrowth either from the remaining ring apophysis or leakage of bone morphogenetic proteins (BMP-INFUSE, Medtronic, Minneapolis, Minnesota) below the traversing nerve root (Supplemental Video 2; http://www.ijssurgery.com/look up/suppl/doi:10.14444/5016/-/DC1/Video2.mp4). Only 1 predominant zone of foraminal stenosis was assigned per patient, and the neuroforaminal height was documented rather than the extent of interbody fusion cage subsidence to stratify analysis. When present, disc herniations involving the lateral recess were grouped as either extruded or contained.

\section{Surgical Techniques}

All surgical procedures employed the endoscopic transforaminal approach using the "outside-in" technique in which the working sheath is placed into the lower portion of the neuroforamen, thus retracting and avoiding the exiting nerve root. No part of the cannula tip or the endoscope is positioned in the disc space. The surgical technique used by the author (originally popularized by 


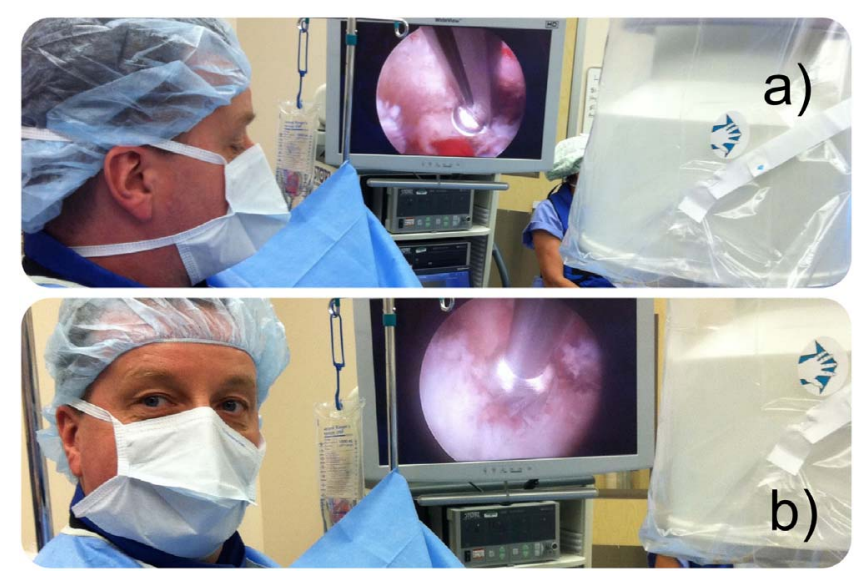

Figure 6. Lumbar endoscopic transforaminal decompression surgery here is shown with an endoscopic Kerrison rongeur (a) and power drill (b) that is advanced through the central working channel of the endoscope.

Hoogland et al. ${ }^{7}$ and Schubert and Hoogland ${ }^{8}$ ) employs a foraminoplasty in patients with or without lateral stenosis for the treatment of the herniated disc (Figures 5 and 6).

Procedures were performed in a prone position under local anesthesia and sedation in all patients. In some instances, where access to the L5/S1 neuroforamen was difficult due to a high riding ilium, patients were positioned in the lateral decubitus position. Techniques to define the skin entry point and the surgical trajectory have been described in previous literature. ${ }^{9-12}$ Generally, entry points were laterally at 7-9 $\mathrm{cm}$ at the L3-4 level, 8$10 \mathrm{~cm}$ at the L4-5 level, and 10-12 $\mathrm{cm}$ at the L5-S1 level.

The targeted neuroforamen was accessed as follows: first, an 18-G (150 $\mathrm{mm}$ in length) spinal needle is inserted into the safe zone of Kambin triangle bordered by the traversing nerve root medially, the exiting nerve root laterally, and the lower adjacent pedicle distally. ${ }^{13,14}$ Ideally, the targeting needle was placed on the lateral view into the lower portion of the neuroforamen or into the disc. On the anterior-posterior view, the needle tip should be at the medial interpedicular line. A steel guide wire was then inserted, and the $18-\mathrm{G}$ spinal needle was removed. Dilators, drills, and trephines of increasing diameters were used for foraminal decompression procedures. Additional cannulated reamers measuring 7 and $9 \mathrm{~mm}$ in diameter intended to be used over a guide wire without the protective working cannula were available but rarely used, in order to further minimize risk of dysesthesia of the exiting nerve root and irritation of its dorsal root ganglion.
For the foraminoplasty, bone from the hypertrophied superior and inferior articular process was removed with different instruments including endoscopic chisels, drills, Kerrison rongeurs, and trephines. The endoscopic drills and rongeurs were deployed inside the center-working cannula of the endoscope to lessen the risk of dysesthesia and irritation of the exiting nerve root and its dorsal root ganglion (Figures 5 and 6). In other words, the entire decompression was performed under continuous direct videoendoscopic visualization.

The foraminoplasty was facilitated by changing the trajectory of the instruments to aim for the compressive pathology identified on preoperative studies. In cases of concomitant herniated disc, extruded disc material was removed using forceps and pituitary rongeurs, and contained herniations were decompressed through a small annular window. Epidural bleeding was controlled with a radiofrequency probe (Ellman, Ellman International LLC, Hicksville, New York) under saline irrigation. In cases of previous posterolateral or interbody fusion at the surgical level, neural decompression and dural dissection were facilitated with the use of power tools, similar to a regular foraminal decompression without excessive fibrosis (Supplementary Videos 1 and 2; http://www.ijssurgery.com/lookup/ suppl/doi:10.14444/5016/-/DC1/Video1.mp4; http:// www.ijssurgery.com/lookup/suppl/doi:10.14444/ 5016/-/DC1/Video2.mp4). Fibrotic scar tissue was always dissected starting from the remaining inferior pedicle. Once access into the epidural space past the medial pedicle was gained, the dissection was then taken rostrally by removing any epidural fibrotic tissue of the remaining annulus. This allowed slow dissection of the traversing and exiting nerve roots and the dorsal root ganglion of the latter, thereby reestablishing the axilla and the triangular safe zone between both roots and the inferior pedicle. Bone formation behind an interbody fusion cage or posteriorly dislocated interbody fusion cages were drilled down and removed in a piecemeal fashion with a pituitary rongeur until flush with the floor of the spinal canal (Figures 7 and 8, and Supplementary Video 2; http://www.ijssurgery.com/lookup/ suppl/doi:10.14444/5016/-/DC1/Video2.mp4).

\section{Postoperative Rehabilitation and Use}

Postoperative rehabilitation and supportive care requirements were recorded and analyzed in relation to clinical outcomes with the secondary transfor- 

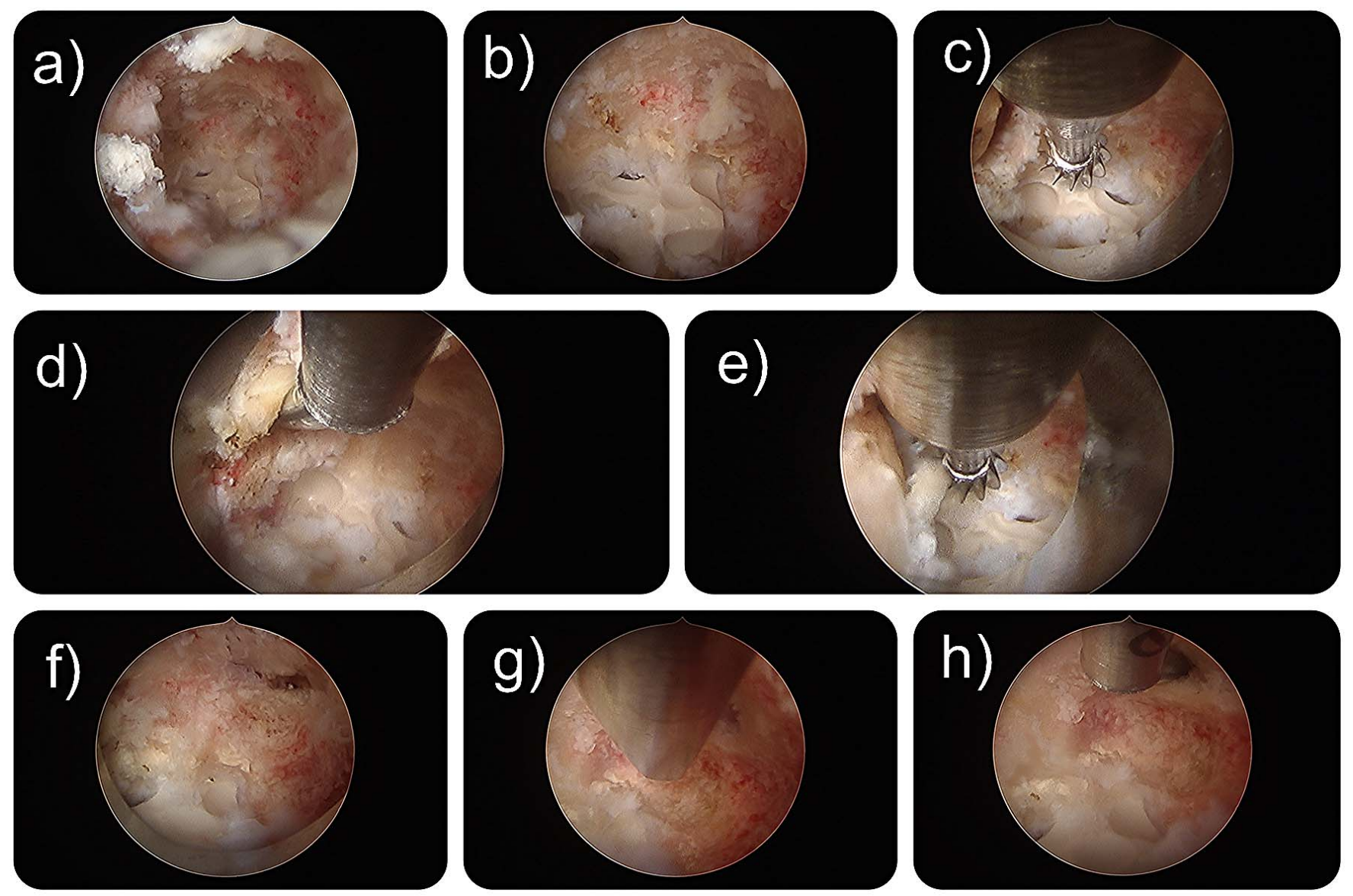

Figure 7. Video-endoscopic photos taken during the transforaminal out-side in endoscopic foraminal and lateral recess decompression in a 76-year-old male patient with multiple previous fusion surgeries. Patient developed heterotopic bone formation behind the interbody fusion cage that was filled with rh-BMP-2 during his index surgery. ( $a$ and b) Creation of an access channel through the posterolateral fusion mass that was also grafted with rh-BMP-2. (c) The PEEK interbody fusion cage is exposed. The lateral recess is accessed in the axilla between the exiting and traversing nerve roots with a probe to confirm decompression. (e) The exiting nerve root is completely decompressed. $(\mathrm{f}-\mathrm{h})$ The lateral recess is decompressed and probed.
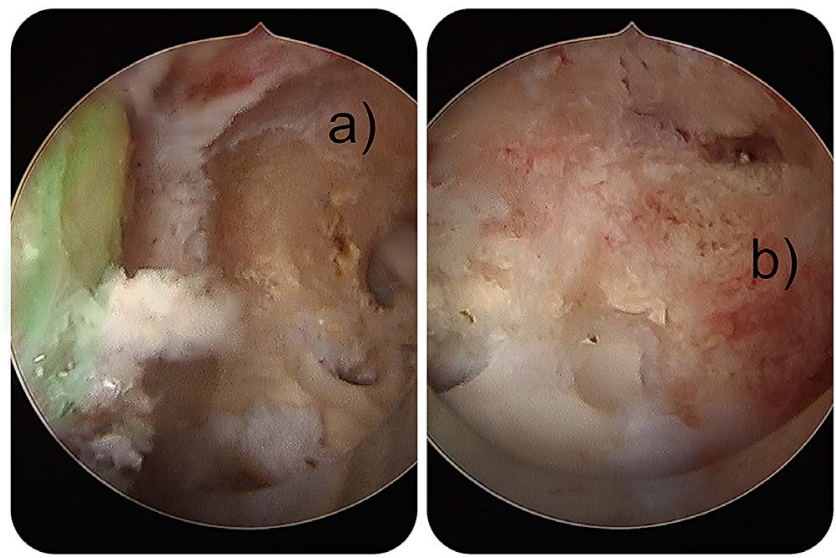

Figure 8. Video-endoscopic photos taken during the transforaminal out-side in endoscopic foraminal and lateral recess decompression in a 76-year-old male patient with multiple previous fusion surgeries. Patient developed heterotopic bone formation behind the interbody fusion cage that was filled with rh-BMP-2 during his index surgery. ( $a$ and $b$ ) Decompression of the exiting (shaded in green in a) and the traversing (b) nerve root. aminal endoscopic decompression procedure in lumbar monoradiculopathy patients due to lateral stenosis with or without herniated disc. During their regular postoperative visits, patients were asked whether they participated in any active exercise programs, physical or occupational therapy, or chiropractic care, or had acupuncture or spinal injection treatments. In addition, the patient's use of nonsteroidal anti-inflammatories, narcotics, and other types of pain medication was recorded. Lastly, patients were asked whether they developed any new pain syndromes or had hitherto unknown conditions that negatively impacted their walking endurance.

\section{Cost Analysis}

A direct estimated cost analysis was performed based on the 2012 Medicare fee schedules for the professional physician fee. Physician reimburse- 


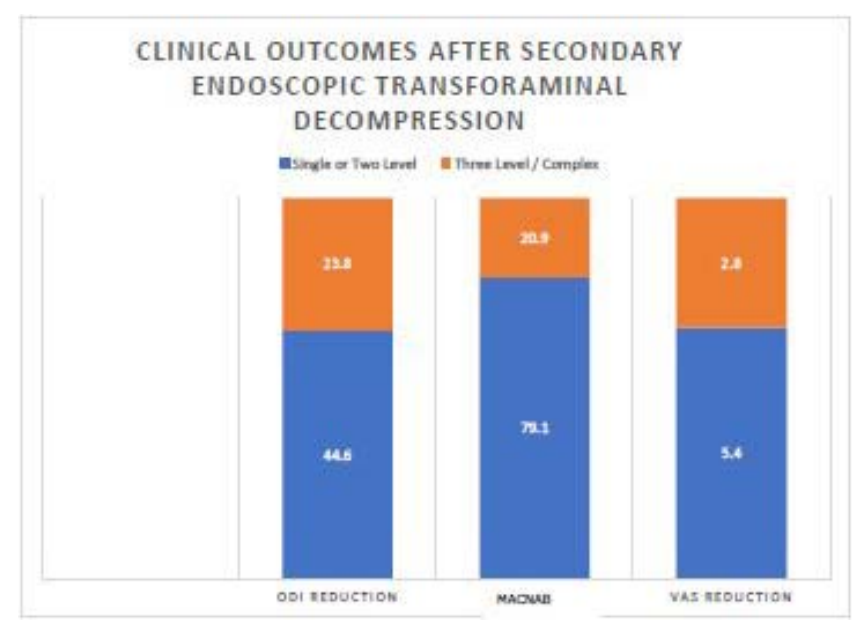

Figure 9. Postoperative ODI (44.6 vs. 23.8) and VAS (5.4 vs. 2.8 ) score reductions were much greater in patients with single- or 2-level secondary surgeries, respectively. Most patients $(79.1 \%)$ with single- to 2-level secondary surgeries also had "excellent" to "good" outcomes according to Macnab criteria, whereas "fair" to "poor" outcomes were reported almost exclusively by patients with 3-level or complex prior surgeries.

ments for open lumbar laminectomy and instrumented fusion surgery in a hospital were recorded and compared to those for the patient's decompression surgery done in an outpatient ambulatory surgery center. Direct cost savings were calculated on a case-by-case basis by subtracting the direct cost of the physician professional fee in the ambulatory surgery center for the follow-up surgery from the cost of the patient's prior surgery done at a hospital. We attempted unsuccessfully to obtain the direct hospitalization cost of the prior inpatient surgery. Indirect cost savings were calculated on patient reported annual salaries and by self-reported expenditures due to surgery-related postoperative use. Savings were estimated by calculating the difference between the number of days prior to return to work reported by patients who worked before and after their primary inpatient, as well as their secondary outpatient, endoscopic transforaminal decompression (27 of 48).

\section{Statistical Methods and Cost Analysis}

For the clinical outcomes analysis, cross-tabulation statistics and measures of association were computed for 2-way tables using IBM SPSS Statistics, version 15.0. Using patient satisfaction data and clinical outcomes data based on the modified Macnab criteria, VAS, foraminal zone classification, MRI classification of disc degeneration, definition of the location of any herniated disc, and foraminal height and width parameters as row and column variables, and age (older than 50 years and younger than 50 years) as a control variable (layer factor), the cross-tabulation procedure was employed to form 1 panel of associated statistics and measures for each value of the layer factor (or a combination of values for 2 or more control variables). This correlation matrix allowed the calculation of variable combinations, if no association was found between clinical outcome and variable distribution was equal. Both the Pearson $\chi^{2}$ and the likelihood-ratio $\chi^{2}$ tests were used as statistical measures of association.

\section{RESULTS}

Of the 258 patients who underwent outpatient decompression from 2010 through 2013, 48 patients had previous spinal surgery prior to their endoscopic transforaminal surgery. As shown in Figure 9, secondary surgery was commonly performed at the L4-5 segment (26 levels; $54.1 \%$ ), followed by L5-S1 (14 levels; 29.2\%), L3-4 (7 levels; 14.6\%), and L2-3 (1 level; $2.1 \%$ ). At final follow-up, patients with single-level and 2-level prior surgeries reported an average ODI reduction following their secondary surgery of $44.6 \%$, with an average final score of 14.8. Less favorable ODI score reductions following secondary surgery $(23.8 \%)$ were reported by patients who had more than 2-level or complex prior multilevel surgery. According to the modified Macnab criteria, "excellent" and "good" results were obtained with the secondary surgery in $79.1 \%$ (38 of 48) of patients with no more than a single 1or 2-level prior lumbar surgery. In this group, the mean VAS score decreased from $7.7 \pm 1.8$ preoperatively to $2.3 \pm 1.1$ at final follow-up $(P<.01)$. "Fair" and "poor" results with the secondary surgery were seen in $20.9 \%$ (10 of 48) of patients with several prior surgeries or complex multilevel previous lumbar surgeries (Figure 10). In this subgroup, the VAS score reduction was also minimal, from $8.1 \pm 2.3$ preoperatively to $5.3 \pm 1.7$ at final follow-up.

Patients with ongoing postoperative sciatica after previous lumbar laminectomy surgery (22 of 48 patients) were treated for neuroforaminal stenosis in the exit (14 of 22), middle (5 of 22), and entry ( 3 of 22) zones at the same levels of the previous index surgery. Foraminal stenosis was caused by reduction of neuroforaminal height due to disc degeneration (Pfirmann grades III-V) ${ }^{5}$ and facet hypertrophy with subluxation of the superior 


\section{SECONDARY SURGERY BY LEVEL}

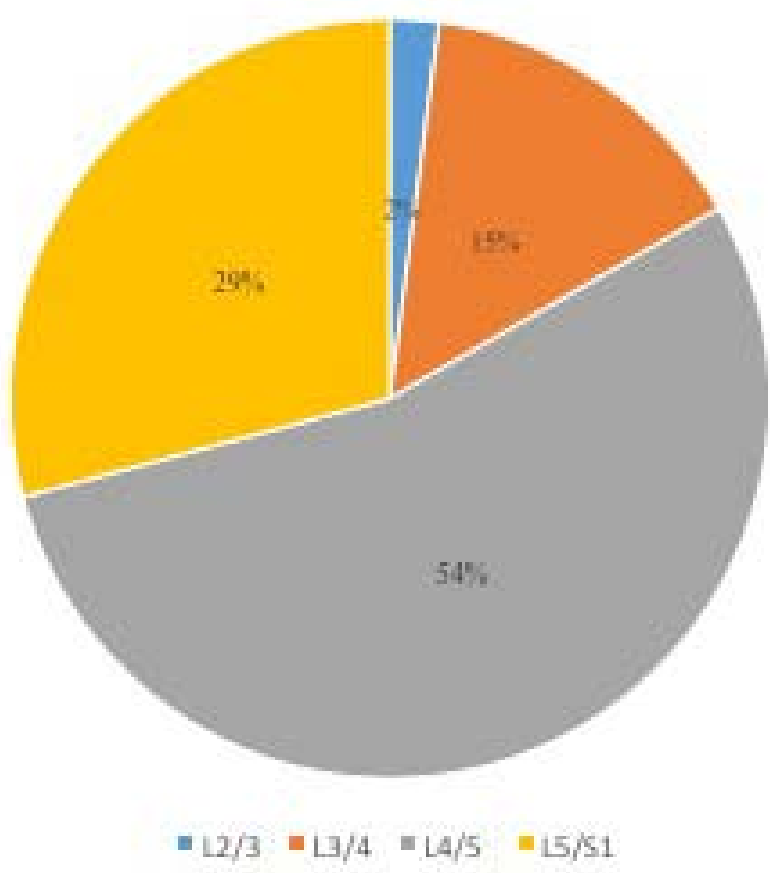

Figure 10. Secondary surgery by lumbar level via endoscopic transforaminal decompression surgery.

articular process into the axilla between the exiting and traversing nerve root. In a group of 26 patients who had prior fusion surgery, 19 patients had surgery for symptomatic adjacent segment disease and were essentially treated in the same manner as patients with prior laminectomy. The remaining 7 patients had undergone secondary surgery performed for symptomatic nerve root entrapment within the surgically fused levels. In the fusion group patients with adjacent segment disease, stenotic lesions were treated in the exit (6 of 19), middle (9 of 19), and entry (4 of 19) zones, respectively. The remaining 7 fusion patients had nerve root entrapment caused by posterior interbody fusion cage migration or subsidence (3 patients), both typically causing nerve root compression from below the traversing nerve root, and overgrowth of the posterolateral fusion mass in another 4 patients who underwent an open decompression instrumented fusion with placement of bone graft into the posterolateral gutter.

Postoperative complications were limited to irritation of the dorsal root ganglion, which occurred in $25 \%$ (12 of 48 ) of patients. There were no wound infections or admissions to a hospital for further postoperative care. All patients with "excellent" and "good" outcomes measured by modified
Macnab criteria, who were working before and after the primary and secondary surgery (27 of 38), reported earlier return to work after the endoscopic outpatient surgery $(2.6 \pm 0.8$ weeks $)$ than with the prior inpatient open spinal surgery $(8.1 \pm 4.5)$.

Based on the 2012 Medicare fee schedule for physician professional fees, the average direct cost created by physician payments was determined to be \$2199.92 per case for the primary hospital-based laminectomy surgery, and $\$ 5135.35$ for the primary hospital-based laminectomy and instrumented fusion surgery. These numbers include single-level and multilevel surgeries. The average direct cost of $\$ 1551.72$ per case created by physician professional fees for the secondary outpatient transforaminal lumbar decompression surgery was also estimated based on the 2012 Medicare fee schedule. The estimated average cost savings for all cases by performing the secondary decompression surgery in an ambulatory outpatient surgery center setting rather than in a hospital was \$2265.68: a 40.6\% reduction. The estimated direct cost savings due to increased physician reimbursement for fusions by performing the outpatient decompression rather than a revision of the previous fusion becomes even more apparent when breaking the figure down by number of levels previously fused: Single-level fusion $=\$ 3271.75$, 2-level fusion $=\$ 3854.21$, and 3 -level fusion $=\$ 5054.99$. Direct cost from hospitalbased expenditures could not be calculated because no access to that information was available.

Indirect cost savings were estimated based on the average loss of patient self-reported annualized income from missed work days in the subgroup of 27 of 48 patients, who were gainfully employed before and after the primary surgery, as well as before and after the secondary spinal surgery. In addition, surgery-related postoperative use costs were also recorded. The average indirect cost from lost salary due to the primary inpatient surgery was estimated to be $\$ 17245.68$, and from the secondary outpatient surgery it was estimated to be $\$ 6392.07$. Thus, performing the secondary surgery in an outpatient ambulatory surgery center realized an average cost savings of $\$ 10853.61$ per surgery, equaling a $37.1 \%$ reduction of indirect cost to the patient. Estimated patient indirect cost savings for surgery-related postoperative use expenses (\$ primary surgery $-\$$ secondary surgery) were, on average, $\$ 930.37$ per case. 


\section{DISCUSSION}

This study shows that it is feasible to perform secondary outpatient transforaminal lumbar decompression surgery in patients who have persistent or recurrent symptoms following either a primary lumbar inpatient open laminectomy, or laminectomy and fusion surgery. Most patients enrolled in this study underwent primary lumbar surgery years before in a hospital setting as inpatients, and it seems obvious that technologic advances with endoscopic lumbar decompression systems, together with a general shift from inpatient to outpatient spinal surgery, are the primary motivators for this development. More advanced endoscopes and decompression tools developed within the last 5 years, such as reamers, trephines, motorized shavers, chisels, and rongeurs, afford better visualization and tissue dissection, as well as more effective decompression maneuvers that were hitherto not possible. One example of this trend is the development of more specialized, longer or shorter spinal endoscopes with larger central working channels in various oval or round configurations ranging from 4.1 to $6.9 \mathrm{~mm}$ (or larger) inner working channel diameters that are specifically designed for the transforaminal or direct posterior interlaminar approach, along with more robust optical, irrigation, and suction systems that can tolerate the abuse caused by more excessive bleeding; debridement; hammering; vibration; and vigorous repetitive, high-turnover cleaning and sterilization cycles. Coupled with various optical viewing angles these modern and contemporary endoscopes are much more user-friendly and tailored to individual surgeon requirements, thus opening the path for expanded surgical indications. The latter is clearly demonstrated by the present study and, to the author's best knowledge, the use of endoscopic transforaminal decompression procedure as a secondary surgery to treat recurrent or persistent pain following a failed primary lumbar surgery, whether laminectomy, or laminectomy with fusion, has not been reported.

Another novel aspect of the use of endoscopic transforaminal decompression surgery for revision of failed primary lumbar surgeries lies in its simplicity. It is an elegant method to treat the patient's persistent or new lumbar radiculopathy. Rather than taking the patient to a hospital to carry out an open revision inpatient lumbar laminectomy, or decompression fusion surgery, the secondary decompression procedure is done in an outpatient setting with reduced cost, better patient acceptance, and favorable clinical outcomes. Although not formally analyzed in this feasibility study, patient satisfaction with outpatient surgery has been reported as being higher than when the same surgery is done in an inpatient setting. Patient acceptance of a smaller, targeted outpatient procedure carried out through smaller incisions is typically higher, presumably because of a lower amount of blood loss, fewer complications, earlier narcotic independence from surgery, and earlier return to desired activities and to work.

Essentially all patients, regardless of whether they had undergone prior laminectomy or prior laminectomy and instrumented fusion surgery, were treated for lateral recess stenosis. Persistent symptoms without ever having had a period of pain relief from the primary surgery implied incomplete decompression of the lateral recess and/or neuroforamina during the primary surgery. Recurrence of symptoms after some time of pain relief following a laminectomy implied progression of the underlying lumbar degenerative process, where progressive loss of disc height at the same surgical or adjacent levels after laminectomy may produce recurrent symptomatic lateral recess and foraminal stenosis. In many cases (19 of 26 patients) recurrence of symptoms in the lumbar fusion patient subgroup was due to adjacent segment disease, where lateral recess and foraminal stenosis had formed either proximally or distally to a primary instrumented fusion.

Only 7 patients from the entire group of 48 secondary surgery patients had a transforaminal endoscopic decompression surgery within the spinal fusion to treat problems caused either by a displaced or subsided interbody fusion cage (typically traversing nerve root compression syndrome), or by overgrowth of the posterolateral fusion mass (exiting nerve root compression syndrome). In other words, 41 of the 48 secondary surgery patients underwent outpatient endoscopic transforaminal decompression surgery under circumstances that were no different from other primary outpatient endoscopic transforaminal decompression surgeries, and the author of the present article essentially performed the same surgery using similar techniques as would have been done in patients undergoing this procedure as a primary surgery in an ambulatory surgery center setting. Given "excellent" and 
"good" outcomes in most failed primary lumbar surgery patients following the secondary procedure, this study concludes that having had prior lumbar surgery does not constitute a contraindication to the transforaminal endoscopic outpatient decompression surgery. In fact, expected outcomes should be similar to a situation in which the patient had no prior lumbar surgery., 45,16

Advanced surgical skills, however, are required to deal with recurrent or persistent nerve root entrapment within a spinal fusion surgery. Displaced or subsided interbody fusion cages as well as overgrown posterolateral fusion can create volumetric reduction of the neuroforamen and lateral recess, and thereby cause symptoms. Although clinical Macnab outcomes with the secondary transforaminal decompression surgery in the patients who previously underwent an operation or patients with prior complex lumbar multilevel laminectomy fusion surgery were less favorable, these patients still improved, and the author would submit that clinical outcomes with open revision decompression with or without the inclusion of instrumented fusion may be less favorable as well.

As sciatica and neurogenic claudication was the main reason for surgical intervention, reduction of leg pain was analyzed using a VAS for leg pain as one of the primary outcome measures. There was significant improvement in the VAS and the clinical outcome at final follow-up, suggesting that the outpatient transforaminal endoscopic decompression procedure is effective in most patients who had undergone previous primary lumbar surgery. In the present study, the clinical outcomes as measured by the ODI and modified Macnab criteria are comparable to success rates reported by patients undergoing laminectomy for spinal stenosis. ${ }^{15-17}$

The importance of preoperative planning of transforaminal endoscopic removal of herniated discs has been stressed by Lee et al., ${ }^{4}$ who suggested a classification based on the location of a migrated disc fragment. According to preoperative sagittal MRI images, they defined 4 zones depending on the direction and distance from the disc space: zone 1, from the inferior margin of the upper pedicle to 3 $\mathrm{mm}$ below of the inferior margin of the upper pedicle; zone 2, from $3 \mathrm{~mm}$ below of the inferior margin of the upper pedicle to the inferior margin of upper vertebral body; zone 3, from the superior margin of the lower vertebral body to the center of the lower pedicle; and zone 4, from the center to the inferior margin of the lower pedicle.

In this study, previously published radiographic classification systems ${ }^{3-5}$ were employed in the preoperative decision-making in patients with symptomatic foraminal stenosis, which was shown to correlate with clinical outcomes according to the modified Macnab criteria. ${ }^{2}$ In 1988, Lee et al. ${ }^{3}$ identified a 3-zone classification system of the neuroforamen by dividing it into entry, middle, and exit zones. Moreover, the height of the neuroforamen underneath the articular processes of the lumbar facet joint can also be used to classify spinal stenosis. In 1995, Hasegawa et al. ${ }^{6}$ defined the width of the neuroforamen of $5 \mathrm{~mm}$ or more as normal. He suggested that a reduced width of 3 to $4 \mathrm{~mm}$ is suggestive of spinal stenosis and that a width of 2 $\mathrm{mm}$ or less is associated with nerve root compression approximately $80 \%$ of the time. The classification systems were successfully employed, as reported in previously published studies, and their use was shown to correlate with favorable clinical outcomes. $^{18}$

This study also showed that application of radiographic grading systems of foraminal stenosis may assist in selecting appropriate surgical candidates for the transforaminal decompression procedure regardless of whether the patient had prior lumbar surgery. The same principles apply to employing these classification systems to preoperative patient selection and preoperative planning, as well as stratifying patients for appropriate surgical indications. Furthermore, the present study also showed that contemporary lumbar endoscopic decompression systems can allow more sophisticated endoscopic decompression surgeries in skilled hands.

Besides comparable clinical outcomes with the lumbar transforaminal endoscopic decompression surgery in patients who previously underwent operation, this surgical technique bears the upside of additional direct and indirect cost savings that can be realized by performing the surgery in an outpatient surgery center rather than in a hospital setting, where the costs of admitting the patient are higher by far. Hospitalization may further increase cost through greater postoperative complication rates due to higher rates of hospital-acquired wound infection and urinary tract infection, as well as pneumonias. Additional problems may arise in the hospital from medication errors and deviation from 
the postoperative rehabilitation protocol because the surgeon may not have complete control over custody of the patient.

There are, however, some limitations of the present study. These are primarily related to the small number of patients (48) with failed primary lumbar spinal surgery who eventually underwent secondary outpatient surgery via the transforaminal approach. This may be due to several reasons. First, failure of the primary lumbar procedure is not commonplace but does occur in a small subset of patients (i.e., the reported number of patients presenting with this problem was small). Second, the endoscopic transforaminal approach is still only performed in select centers by few surgeons, perhaps in part because of the steep learning curve, although it has gained significant recognition in the last few years. Finally, the combination of these 2 factors only allowed the accumulation of a small number of patients within this study because all secondary outpatient surgeries were completed in a singlesurgeon practice setting, and patients from other surgeons who met these criteria could not be easily recruited. Therefore, the small number of patients included in this study only allowed the cross tabulation of a limited number of categories against covariates. For example, analysis of outcomes with the transforaminal decompression within the patients who previously underwent multiple operations, or in patients with complex or multilevel surgeries with or without fusion, was not possible because meaningful clinical differences in the statistical analysis could simply not be identified.

Additional limitations relate to the cost savings analysis. This retrospective case cohort study (level III clinical evidence) was not set up to perform a detailed direct and indirect cost analysis in terms of quality-adjusted-life-years savings measured in actual dollar amounts. Instead, cost savings analysis was limited to estimating direct cost savings by assuming that all patients were Medicare beneficiaries rather than recording the cost under their individual insurance plan for the years of their primary surgery and then secondary surgery, or analyzing the respective explanation of benefit statements for each individual patient. These data were simply not available for all patients, particularly not for the primary lumbar surgery patients because most of these surgeries had been performed by other surgeons some years before. Similarly, indirect costs were only estimated in the subset of patients who were working both before the primary and before the secondary surgery. These calculations were based on patient self-reported annual incomes and the number of work days missed because of having open primary lumbar surgery versus secondary outpatient transforaminal decompression surgery. Other additional costs that patients may have incurred because of engaging in other self-directed treatments, such as acupuncture, massage or chiropractic therapy, or homeopathic and alternative medical management, may also have been incomplete. Although these methods of estimating direct and indirect costs have been used, the calculated cost savings in the present study are merely an estimate and most likely not an accurate representation of the true direct and indirect costs. Presumably, the difference between estimated and true direct and indirect costs is conceivably higher. However, the cost savings with performing a secondary targeted lumbar decompression surgery in an outpatient surgery center rather than in a hospital are overwhelming, with a $40.6 \%$ reduction in cost.

\section{CONCLUSION}

This study shows that outpatient transforaminal endoscopic decompression surgery should be considered as a viable alternative to inpatient open revision lumbar surgery as a means of providing pain relief with a secondary surgery once the primary surgery has failed. This conclusion is based on the merits of favorable clinical results that at a minimum seem comparable to those of open decompression, and on the merits of lower complication rates. Moreover, cost savings in the context of value-based health care that are mandated by patients, insurance providers, governmental institutions, and review boards will afford the spinal surgeon a modern platform to better position himself or herself competitively in the dynamically changing health care environment. Further study of the merits of this technique seems warranted in a larger patient population.

\section{REFERENCES}

1. Sengupta DK, Herkowitz HN. Lumbar spinal stenosis: treatment strategies and indications for surgery. Orthop Clin North Am. 2003;34(2):281-295.

2. Macnab I. Negative disc exploration: an analysis of the causes of nerve-root involvement in sixty-eight patients. $J$ Bone Joint Surg Am. 1971;53(5):891-903. 
3. Lee CK, Rauschning W, Glenn W. Lateral lumbar spinal canal stenosis: classification, pathologic anatomy and surgical decompression. Spine (Phila Pa 1976). 1988;13(3):313-320.

4. Lee S, Kim SK, Lee SH, et al. Percutaneous endoscopic lumbar discectomy for migrated disc herniation: classification of disc migration and surgical approaches. Eur Spine $J$. 2007;16(3):431-437.

5. Pfirrmann CW, Metzdorf A, Zanetti M, Hodler J, Boos N. Magnetic resonance classification of lumbar intervertebral disc degeneration. Spine (Phila Pa 1976). 2001;26(17):18731878 .

6. Hasegawa T, An HS, Haughton VM, Nowicki BH. Lumbar foraminal stenosis: critical heights of the intervertebral discs and foramina: a cryomicrotome study in cadavera. $J$ Bone Joint Surg Am. 1995;77(1):32-38.

7. Hoogland T, Schubert M, Miklitz B, Ramirez A. Transforaminal posterolateral endoscopic discectomy with or without the combination of a low-dose chymopapain: a prospective randomized study in 280 consecutive cases. Spine (Phila Pa 1976). 2006;31(24):E890-E897.

8. Schubert M, Hoogland T. Endoscopic transforaminal nucleotomy with foraminoplasty for lumbar disk herniation. Oper Orthop Traumatol. 2005;17(6):641-661.

9. Yeung AT, Yeung CA. Minimally invasive techniques for the management of lumbar disc herniation. Orthop Clin North Am. 2007;38(3):363-372.

10. Tsou PM, Alan Yeung C, Yeung AT. Posterolateral transforaminal selective endoscopic discectomy and thermal annuloplasty for chronic lumbar discogenic pain: a minimal access visualized intradiscal surgical procedure. Spine $J$. 2004;4(5):564-573.

11. Tsou PM, Yeung AT. Transforaminal endoscopic decompression for radiculopathy secondary to intracanal noncontained lumbar disc herniations: outcome and technique. Spine J. 2002;2(1):41-48.

12. Yeung AT, Yeung CA. Advances in endoscopic disc and spine surgery: foraminal approach. Surg Technol Int. 2003; 11:255-263.

13. Kambin P, Casey K, O'Brien E, Zhou L. Transforaminal arthroscopic decompression of lateral recess stenosis. J Neurosurg. 1996;84:462-467.

14. Kambin P, O’Brien E, Zhou L. Arthroscopic micro- discectomy and selective fragmentectomy. Clin Orthop. 1998;347:150-167.

15. Kim MJ, Lee SH, Jung ES, et al. Targeted percutaneous transforaminal endoscopic diskectomy in 295 patients: comparison with results of microscopic diskectomy. Surg Neurol. 2007;68(6):623-631.

16. Ahn Y, Lee SH, Park WM, Lee HY, Shin SW, Kang HY. Percutaneous endoscopic lumbar discectomy for recurrent disc herniation: surgical technique, outcome, and prognostic factors of 43 consecutive cases. Spine (Phila Pa 1976). 2004;29(16):E326-E332.

17. Fokter SK, Yerby SA. Patient-based outcomes for the operative treatment of degenerative lumbar spinal stenosis. Eur Spine J. 2006;15(11):1661-1669.

18. Lewandrowski KU. "Outside-in" technique, clinical results, and indications with transforaminal lumbar endoscopic surgery: a retrospective study on 220 patients on applied radiographic classification of foraminal spinal stenosis. Int $J$ Spine Surg. 2014;8.

Disclosures and COI: The author does not have any conflicts of interest associated with this study.

Corresponding Author: Kai-Uwe Lewandrowski, MD, Center for Advanced Spine Care of Southern Arizona, 4787 East Camp Lowell Drive, Tucson, AZ 85712. Phone: (520) 204-1495; Fax: (623) 218-1215; Email: business@tucsonspine. com.

Published 3 August 2018

This manuscript is generously published free of charge by ISASS, the International Society for the Advancement of Spine Surgery. Copyright @ 2018 ISASS. To see more or order reprints or permissions, see http://ijssurgery.com. 